\title{
COMPASS Results on the Strange Quark Polarisation
}

\author{
Helena Santos, on behalf of the COMPASS Collaboration \\ LIP - Laboratório de Instrumentação e Física Experimental de Partículas \\ Av. Elias Garcia, 14, 1000-149, Lisboa, Portugal
}

\begin{abstract}
A main topic of investigation at the COMPASS experiment is the spin structure of the nucleon. This article reports on the experimental procedure to measure the polarisation of the strange quarks in polarised deep inelastic scattering, both in inclusive and semiinclusive reactions. The dependence of the results for the strange quark polarisation on the fragmentation functions is discussed.
\end{abstract}

\section{Experimental Procedure}

COMPASS makes use of the CERN-SPS facilities, impinging a high intensity $160 \mathrm{GeV}$ muon beam on a ${ }^{6} \mathrm{LiD}$ polarised target. The detailed description of the spectrometer can be found at Ref. [2]. Data presented in this article have been collected in the years 2002, 2003, 2004 and 2006 .

\section{The $g_{1}^{N}$ structure function}

In order to access the spin-dependent structure function, $g_{1}^{d}$, the longitudinal photondeuteron asymmetry, $A_{1}^{d}$, has to be evaluated. In the framework of the quark parton model this quantity can be directly related to the quark polarisation, $\Delta q$, via

$$
A_{1}=\frac{\left(\sigma_{\gamma \mu}^{\uparrow \downarrow}-\sigma_{\gamma \mu}^{\uparrow \uparrow}\right)}{\left(\sigma_{\gamma \mu}^{\uparrow \downarrow}+\sigma_{\gamma \mu}^{\uparrow \uparrow}\right)} \simeq \frac{\sum_{q} e_{q}^{2}(\Delta q+\Delta \bar{q})}{\sum_{q} e_{q}^{2}(q+\bar{q})}
$$

where the arrows indicate the relative beam and target spin orientations. Events are selected by cuts on the four-momentum transfer squared, $Q^{2}>1(\mathrm{GeV} / c)^{2}$, and the fractional energy of the virtual photon, $0.1<y<0.9$. After data selection, $89 \times 10^{6}$ events are distributed over 15 bins of the Bjorken scaling varible, in the range $0.004<x<0.7$. The left panel of Fig. 1 shows $A_{1}^{d}$ as a function of $x$, as measured by COMPASS using 2002, 2003 and 2004 data [3]. The asymmetry is 0 for $x<0.05$ and gets larger as $x$ increases, reaching $60 \%$ at $x \simeq 0.7$. The error bars are the statistical ones and the grey band corresponds to the systematic errors, whose sources come from the uncertainty on the beam and the target polarisations $(5 \%$ each), the dilution factor (6\%) and the depolarisation factor (4-5\%). Radiative corrections and the neglect of the transverse asymmetry $A_{2}$ are found to have a small effect. The upper limit for the systematic error due to false asymmetries is half of the statistical one.

The spin-dependent structure function of the nucleon, $g_{1}(x)$, is obtained from $A_{1}(x)$ and the spin-independent structure function $F_{2}(x)$ through

$$
g_{1}(x)=A_{1}(x) \frac{F_{2}(x)}{2 x(1+R)},
$$

DIS 2009 

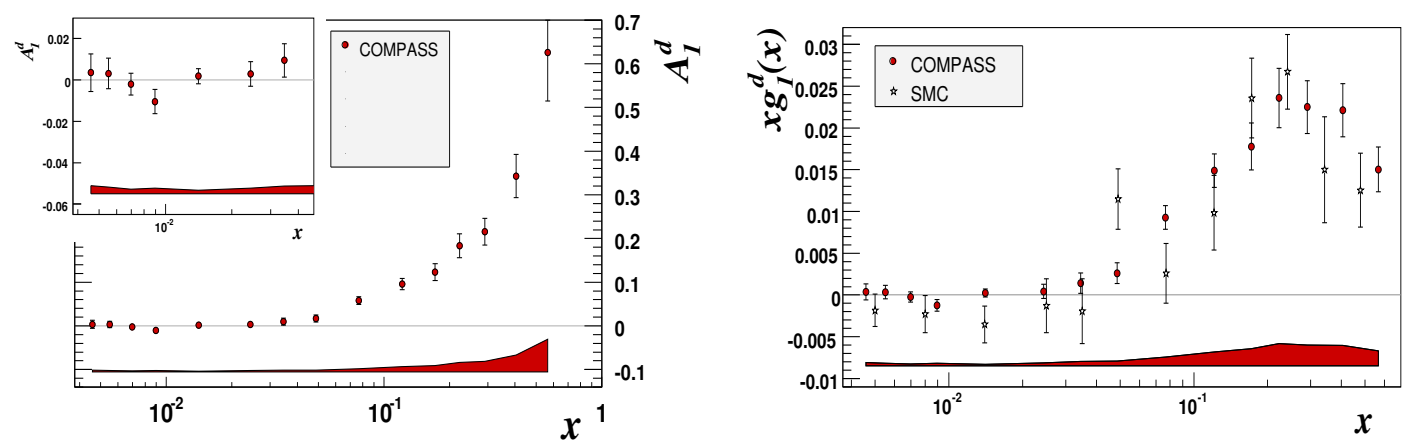

Figure 1: Left: The asymmetry $A_{1}^{d}(x)$ as a funtion of $x$. The error bars are the statistical ones. The band shows the systematic errors. Right: The spin-dependent structure function of the deuteron, $g_{1}^{d}$, as a function of $x$. The errors bars are the statistical ones. The band shows the COMPASS systematic errors.

where $R$ is the ratio of the longitudinal to transverse photon absorption cross-sections. The right panel of Fig. 1 shows $g_{1}^{d}$ as a function of $x$ [3]. The SMC results [4] have been evolved to the $Q^{2}$ of the corresponding COMPASS points. We have calculated the integral of $g_{1}^{N}$ using exclusively the experimental values of COMPASS evolved to $\mathrm{Q}_{0}^{2}=3 \mathrm{GeV}^{2}$ and averaged over two fits [3]. Taking into account the contributions $(2 \%)$ from the fits in the unmeasured regions of $x<0.004$ and $x>0.7$, we obtain:

$$
\Gamma_{1}^{N}\left(Q^{2}=3(\mathrm{GeV} / c)^{2}\right)=0.050 \pm 0.003 \text { (stat.) } \pm 0.003 \text { (evol.) } \pm 0.005 \text { (syst.). }
$$

The second error accounts for the difference in $Q^{2}$ evolution between the two fits. The systematic error is the dominant one and mainly corresponds to the uncertainty of the beam and the target polarisations and of the dilution factor. $\Gamma_{1}^{N}$ is related to the matrix element of the singlet axial current $a_{0}$, which measures the quark spin contribution to the nucleon spin. The relation between $\Gamma_{1}^{N}$ and $a_{0}$, independent on $Q^{2}\left(\hat{a}_{0}=a_{0\left(Q^{2} \rightarrow \infty\right)}\right)$ (Ref. [5]), is

$$
\Gamma_{1}^{N}\left(Q^{2}\right)_{Q^{2} \rightarrow \infty}=\frac{1}{9} \hat{C}_{1}^{S}\left(Q^{2}\right) \hat{a}_{0}+\frac{1}{36} C_{1}^{N S}\left(Q^{2}\right) a_{8} .
$$

The coefficients $\hat{C}_{1}^{S}$ and $C_{1}^{N S}$ have been calculated in perturbative QCD up to the third order in $\alpha_{s}\left(Q^{2}\right)$ [5]. From the COMPASS result of Eq. 1 and taking the value of $a_{8}$ measured in hyperon $\beta$ decay, assuming $S U(3)_{f}$ flavour symmetry $\left(a_{8}=0.585 \pm 0.025[6]\right)$, one obtains:

$$
\hat{a}_{0}=0.33 \pm 0.03 \text { (stat.) } \pm 0.05 \text { (syst.). }
$$

with the value of $\alpha_{s}$ evolved from the PDG value $\alpha_{s}\left(\mathrm{M}_{\mathrm{z}}{ }^{2}\right)=0.1187 \pm 0.005$. Combining this value with $a_{8}$, the first moment of the strange quark distribution is:

$$
(\Delta s(x)+\Delta \bar{s}(x))_{Q^{2} \rightarrow \infty}=\frac{1}{3}\left(\hat{a}_{0}-a_{8}\right)=-0.08 \pm 0.01(\text { stat. }) \pm 0.02(\text { syst. }) .
$$

One should keep in mind that the data have been evolved to a common $Q^{2}$ through a NLO fit, whereas the coefficients $\hat{C}_{1}^{S}$ and $C_{1}^{N S}$, as well as $\alpha_{s}\left(Q^{2}\right)$, have been obtained beyond 
NLO. However, the choice of a value close to the average of $Q^{2}$ of the DIS data is expected to minimise the effect of the evolution in the results of $\hat{a}_{0}$ and $\Delta s(x)+\Delta \bar{s}(x)$ quoted above.

\section{$3 \quad \Delta s$ evaluated in semi-inclusive asymmetries}

Another way to access the spin-dependent structure functions is to study the semi-inclusive asymmetries, $A_{1, d}^{h}$. In addition to the data selection of the inclusive sample, the fraction of the photon energy carried out by the hadrons, $z$, should lie between 0.2 and 0.85 . The momentum range of the hadrons is $10-50 \mathrm{GeV} / \mathrm{c}$. Figure 2 shows the inclusive and charged hadron asymmetries $A_{1}^{d}, A_{1, d}^{\pi^{ \pm}}$and $A_{1, d}^{K^{ \pm}}$as a function of $x$, as measured by COMPASS, including also the data taking of 2006. The $x$ range is between 0.004 and 0.3 The asymmetry is 0 for $x<0.05$ and gets larger as $x$ increases, with exception for $A_{1, d}^{K-}$. The lines are NLO predictions of the DSSV fit [7]. With exception for the $A_{1, d}^{K^{-}}$asymmetry, they agree very well with the COMPASS results.

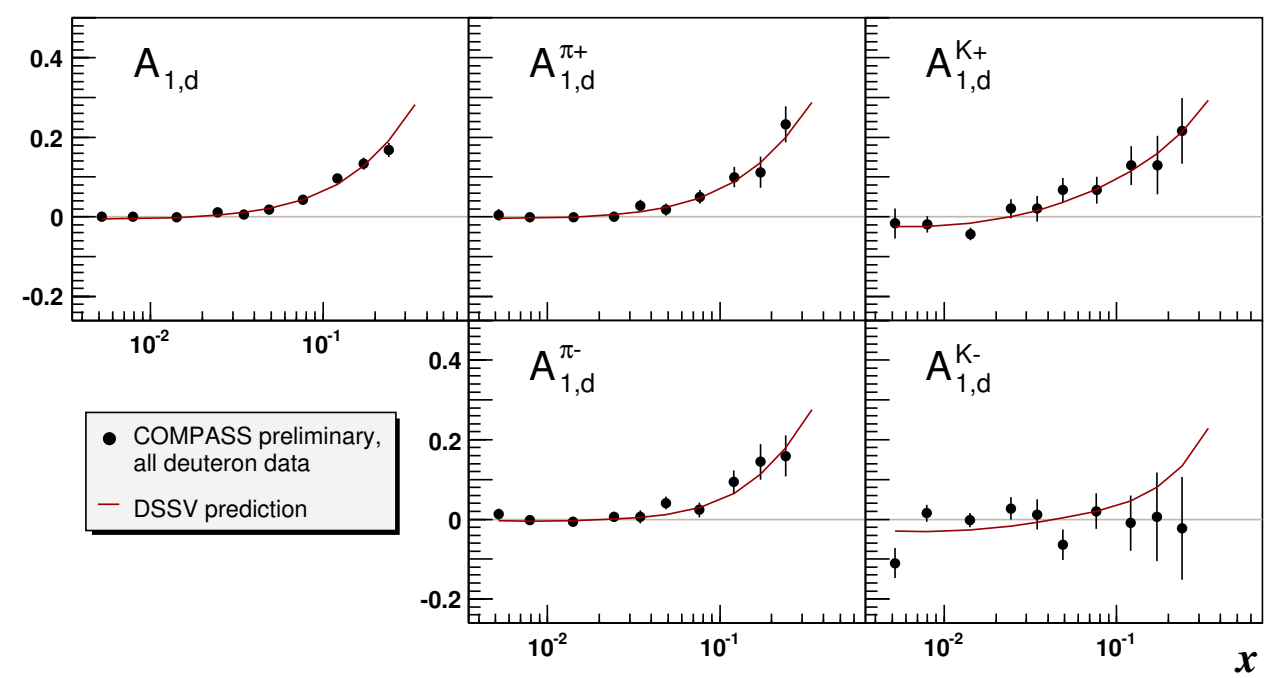

Figure 2: The asymmetries $A_{1}^{d}(x), A_{1, d}^{\pi^{ \pm}}(x)$ and $A_{1, d}^{K^{ \pm}}(x)$ as measured by the COMPASS experiment. The error bars are the statistical ones. The lines are NLO predictions of the DSSV fit [7].

These spin asymmetries are used to determine the polarised parton density functions, $\Delta q\left(x, Q^{2}\right)$ :

$$
A_{1}^{h}\left(x, Q^{2}, z\right)=\frac{\sum_{q} e_{q}^{2}\left(\Delta q\left(x, Q^{2}\right) D_{q}^{h}\left(z, Q^{2}\right)\right)}{\sum_{q} e_{q}^{2}\left(q\left(x, Q^{2}\right) D_{q}^{h}\left(z, Q^{2}\right)\right)},
$$

where $e_{q}$ is the quark charge. For the unpolarised distributions, $q\left(x, Q^{2}\right)$, LO MRST04 [8] are used. The two sets of fragmentation functions, $D_{q}^{h}\left(z, Q^{2}\right)$, are considered from the DSS [9] group and the EMC [10] measurements of the $u$ quark fragmentation into $\pi$ and $K$. The main difference between the two sets is the enhanced $s(\bar{s})$ quark contribution to the production

DIS 2009 
of $K^{-}\left(K^{+}\right)$in DSS, thus only the results on the strange quark polarisation are visibly affected. The fitted polarised PDFs are shown in Fig. 3. The lines are the LO DNS [11] fit to SMC [12] and HERMES [13] data, only. Indeed, the DNS parameterisation predicts successfully our result. The first moments of valence and non-strange quark polarisations,

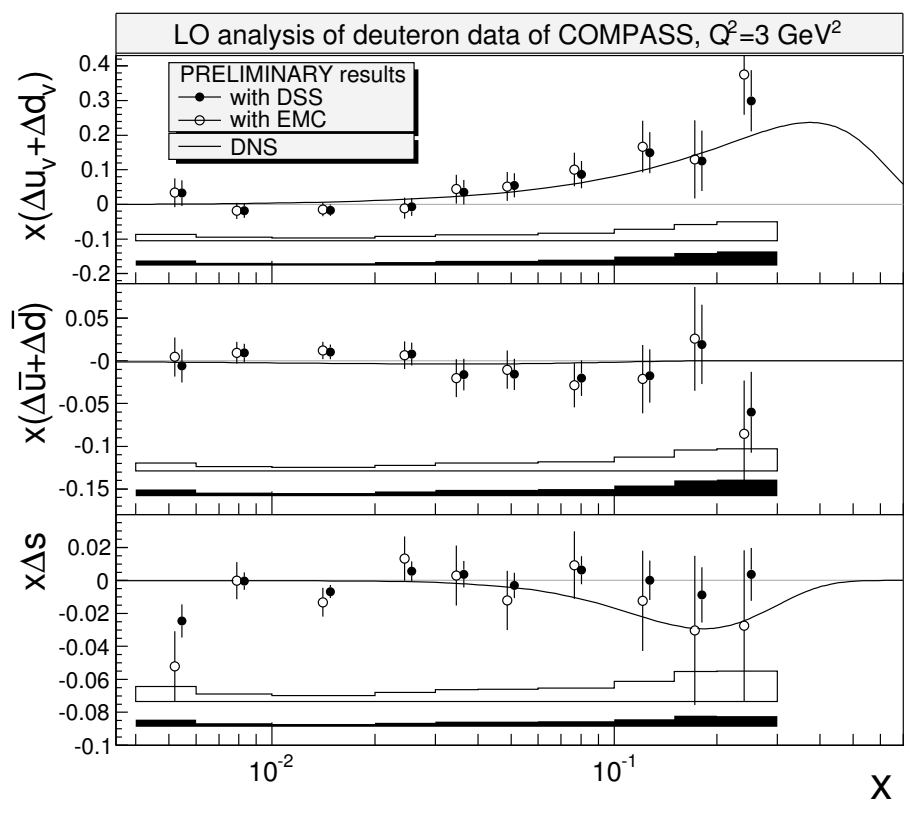

Figure 3: The polarised PDFs for valence, non-strange and strange quarks for two sets of fragmentation functions, evaluated at $Q_{0}^{2}=3 \mathrm{GeV}^{2}$. The error bars are the statistical ones. The bands represent the systematic uncertainties. The curves are the DNS fit which do not include the present COMPASS data.

truncated to the measured $x$ range and obtained with DSS fragmentation functions, are $\Delta u_{v}+\Delta d_{v}=0.28 \pm 0.06 \pm 0.03$ and $\Delta \bar{u}+\Delta \bar{d}=-0.03 \pm 0.03 \pm 0.01$. These values are well in agreement with the ones measured with the difference asymmetry approach [14]. A different scenario concerns the contribution of the strange quark $(\Delta s(S I D I S)=-0.01 \pm 0.01 \pm 0.01)$, from the measured $x$ range) to the nucleon spin, as this SIDIS measurement is not confirmed in inclusive DIS $(\Delta s(D I S)=-0.05 \pm 0.01 \pm 0.01)$, in the full $x$ range, as shown in Sec. 2. A similar disagreement is obtained by HERMES [15].

In order to study the dependence of $\Delta s$ on the fragmentation functions, a different approach is used. In such, $\Delta s$ is related to inclusive and charged kaon asymmetries and to the ratios of strange to favoured, $R_{S F}=\int D_{\bar{s}}^{K+} / \int D_{u}^{K+}$, and unfavoured to favoured, $R_{U F}=\int D_{\bar{d}}^{K+} / \int D_{u}^{K+}$, fragmentation functions. This approach leads to a very similar result on $\Delta s$, as can be seen on the left panel of Fig. 4. The right panel shows the strong dependence of $\Delta s$ on the $R_{S F}$. $R_{U F}$ is fixed to 0.14 from the DSS FF, but large changes of this ratio do not change significantly the result of $\Delta s$. If $R_{S F}>5, \Delta s$ from SIDIS measurements becomes larger than $\Delta s$ obtained in DIS, which implies that $\Delta s$ must be negative in the unmeasured low $x$ range. However, the difference never exceeds $2 \sigma$. More details about this study can be found in [17].

DIS 2009 

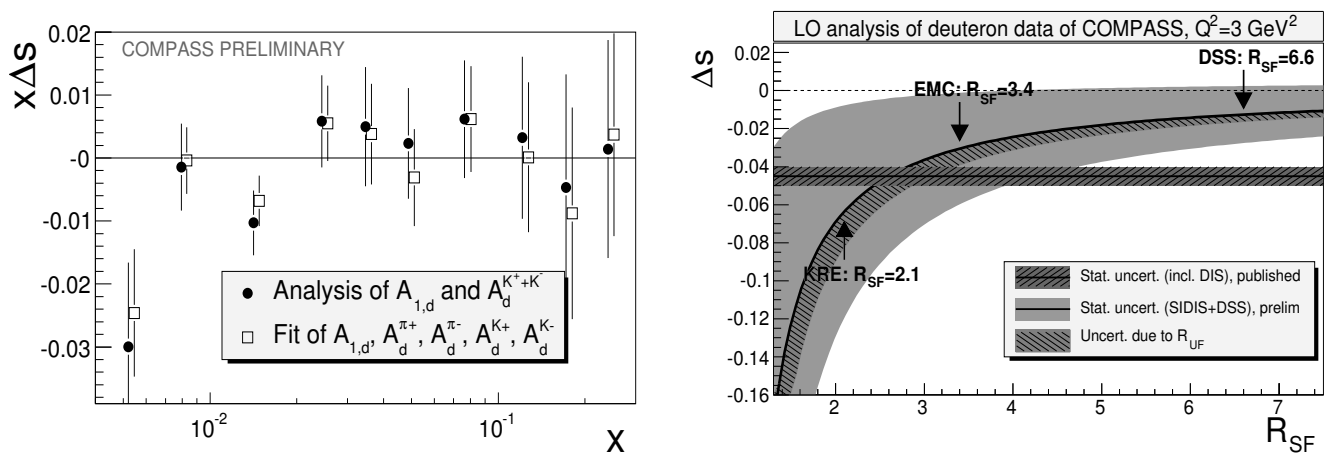

Figure 4: Left: The strange quark helicity distributions derived from inclusive and charged kaon asymmetries compared to the result of the least square fit. Right: Integral of $\Delta s$ as a function of $R_{S F}$ for $R_{U F}$ fixed to 0.14 (thick solid curve). The light grey area is the statistical uncertainty and the hatched band is the statistical uncertainty due to $R_{U F}$. The horizontal band is the full moment of $\Delta s$ derived from COMPASS measurement at DIS. The arrows indicate the values of $R_{S F}$ corresponding to DSS [9], EMC [10] and KRE [16] parameterisations of fragmentation functions.

\section{References}

[1] Slides: http: //indico. cern. ch/contributionDisplay $\cdot$ py? contribId=293\&sessionId=4\&conf Id=53294

[2] P. Abbon et al., (COMPASS Coll.), Nucl. Inst. Meth. A 577455 (2007);

[3] V.Yu. Alexakhin et al. (COMPASS Coll.), Phys. Lett. B 6478 (2007);

[4] B. Adeva et al. (SMC Coll.), Phys. Rev. D 58, 112001 (1998);

[5] S. A. Larin et al., Phys. Lett. B 404, 153 (1997);

[6] Y. Goto et al., Phys. Rev. D 62, 037503 (2003);

[7] D. de Florian, R. Sassot, M. Stratmann, W. Vogelsang, Phys. Rev. Lett. 101072001 (2008);

[8] A. D. Martin, R. G. Roberts, W. J. Stirling, R. S. Thorne, Phys. Lett. B 636259 (2006);

[9] D. de Florian, R. Sassot, M. Stratmann, Phys. Rev. D 75114010 (2007);

[10] A. Arneodo et al., (EMC Coll.), Nucl. Phys. B 321541 (1989);

[11] D. de Florian, G.A. Navarro, R. Sassot, Phys. Rev. D 71094018 (2005);

[12] B. Adeva et al., (SMC Coll.), Phys. Lett. B 420180 (1998);

[13] A. Airapetian et al. (HERMES Coll.), Phys. Rev. D 71012003 (2005);

[14] V.Yu. Alexakhin et al. (COMPASS Coll.), Phys. Lett. B 660458 (2007);

[15] A. Airapetian et al. (HERMES Coll.), Phys. Rev. D 75012007 (2007);

[16] S. Kretzer, Phys. Rev. D 62054001 (2000).

[17] M. Alekseev et al. (COMPASS Coll.), CERN-PH-EP/2009-008, hep-ex/0905.2828, subm. to Phys. Lett. B.

DIS 2009 